Research Paper

\title{
Effects Of Oral Glutamine on Inflammatory and Autophagy Responses in Cancer Patients Treated With Abdominal Radiotherapy: A Pilot Randomized Trial
}

\author{
Juan J. Ortiz de Urbina1,3, Beatriz San-Miguel ${ }^{1}$, Alfonso Vidal-Casariego ${ }^{4}$, Irene Crespo ${ }^{1,2}$, Diana I. \\ Sánchez¹, José L. Mauriz¹,2, Jesús M. Culebras1, Javier González-Gallego1,2, María J. Tuñón¹,2丣 \\ 1. Institute of Biomedicine (IBIOMED), University of León, León, Spain; \\ 2. Centro de Investigación Biomédica en Red de Enfermedades Hepáticas y Digestivas (CIBERehd), Spain; \\ 3. Pharmacy Service, Complejo Asistencial Universitario de León, León, Spain; \\ 4. Endocrinology and Nutrition Service, Complejo Asistencial Universitario de León, León, Spain. \\ $\bowtie$ Corresponding author: mjtung@unileon.es Tel.: +34-987-291261 \\ (1) Ivyspring International Publisher. This is an open access article distributed under the terms of the Creative Commons Attribution (CC BY-NC) license \\ (https://creativecommons.org/licenses/by-nc/4.0/). See http://ivyspring.com/terms for full terms and conditions.
}

Received: 2017.03.24; Accepted: 2017.07.05; Published: 2017.09.04

\begin{abstract}
Background and Aims: Abdominal radiotherapy (RT) causes harm to the mid gastrointestinal mucosa by release of pro-inflammatory cytokines and promotes autophagic changes in tumor cells. This study was aimed to measure the effect of glutamine administration on markers of inflammation and autophagy in cancer patients treated with RT.

Methods: In this double-blind, randomized, controlled pilot trial 43 patients under abdominal RT diagnosed of pelvic or abdominal malignancies receiving glutamine $(30 \mathrm{~g} / \mathrm{d}$ ) or placebo (casein, $30 \mathrm{~g} / \mathrm{d}$ ). Patient recruitment took place in the Complejo Asistencial Universitario of León (CAULE), Spain. Patient evaluation took place at three different time points during the study: before RT (pre-treatment), in the middle of the RT period (mid-treatment), and after finishing RT (post-treatment). Data were compared by analysis of variance and the Newmann Keuls test. Significance was accepted at $p<0.05$.

Results Abdominal RT increased whole blood mRNA levels of inflammatory and autophagic markers, but glutamine administration showed significantly lower expression of toll-like receptor 4 (TLR4), CD36, interleukin-1 $\beta$ (IL-1 $\beta$ ), tumor necrosis factor-alpha (TNF- $\alpha$ ), cyclooxygenase-2 (COX-2), and matrix metalloproteinase-9 (MMP-9). Moreover, glutamine reduced the expression of the transcription factors nuclear factor kappa B (NF- $\kappa B$ ) and activator protein 1 (AP-1). Glutamine also inhibited the autophagic response, with changes in expression of beclin-1, UV radiation resistance associated gene (UVRAG), autophagy-related protein-5 (Atg5), protein 1 light chain 3 (LC3), sequestosome 1 (p62/SQSTM1) and lysosome-associated membrane protein (LAMP)-1.

Conclusions Findings provide evidence that glutamine decreases the inflammatory response and abolishes the changes of the autophagy machinery in patients receiving abdominal RT. The protective effect of glutamine must continue being investigated to disclose further molecular pathways.
\end{abstract}

Key words: glutamine; clinical trial; inflammation; autophagy; radiotherapy.

\section{Introduction}

Inflammatory effects significantly contribute to the disorders associated with abdominal radiotherapy (RT) for malignant diseases. Different studies have shown that after the administration of RT, inflammatory cytokines including IL-1 $\beta$, TNF- $\alpha$, and IL-6, are released from the epithelium and the adjacent connective tissue [1], playing a key role in the development of RT-induced gastrointestinal mucositis [2]. Many inflammatory responses, particularly in the gut, are mediated by the activation of transcription factors nuclear factor kappa B (NF- $\kappa$ B) and activator protein 1 (AP-1) [1]. NF- $\mathrm{BB}$ is a key factor in the inducible expression of many pro-inflammatory genes, which is known to be 
activated by RT and may play a role in the development of radioresistance [3].

Autophagy is an evolutionary conserved response to metabolic stress that recycles cellular proteins and organelles [4] and has a controversial role in relation to stress induced by radiation in tumor cells. Up-regulation of autophagic genes induced by radiation has been considered a cytotoxic mechanism which contributes to cancer cell death [5]. However, it is known that autophagy inhibition increases cancer cell sensitivity to chemotherapy or radiation [6], and it has been reported that the combination of autophagy inhibition with radiation therapy would enhance the efficiency of anti-tumor treatment [7].

Glutamine is ubiquitous, being the main source of amino acids for the intestinal mucosa. Glutamine is largely metabolized by enterocytes and immunocytes [8]. This amino acid has been used in many clinical conditions with high nitrogen losses, trauma, burns, prolonged fasting, chemotherapy, etc. [9]. Glutamine may be beneficial in RT-treated patients. A retrospective research has reported its efficacy in the prevention of acute radiation induced esophagitis [10]. In patients with enteritis due to abdominal and pelvic RT, a previous study has observed lower rates of acute and chronic diarrhea [11], while in a more recent randomized clinical trial no apparent beneficial effects were reported in terms of stool frequency [12]. It is also known that patients with esophageal cancer receiving a combination of omega-3 fatty acids, glutamine, and arginine show reduced levels of inflammatory cytokines [13].

This double blind, placebo controlled pilot study was aimed to investigate whether the use of glutamine prevented the inflammatory response and the autophagic process in cancer patients treated with abdominal radiotherapy.

\section{Methods}

\section{Study patients and experimental design}

Forty three patients $>18$ years with abdominal/pelvic cancer planned to abdominal RT were included. Previous therapeutic actions (surgery, chemotherapy, brachytherapy), were admitted for the trial. Patient recruitment took place in the Complejo Asistencial Universitario of León (CAULE). Exclusion criteria included life expectancy $<1$ year, relevant intestinal diseases (celiac disease, inflammatory bowel disease, Whipple disease), intestinal failure or short bowel syndrome of any etiology, moderate or severe chronic kidney disease, or inability to receive oral medication or to understand the provided information. Included patients signed informed consent. Patients were randomly allocated for double blind pilot study in a 1:1 ratio using computer generated randomization (Figure 1). The randomization code was broken after the completion of statistical analysis. Patient evaluation took place at three different time points during the study: before abdominal RT (pre-treatment), in the middle of the abdominal RT period (mid-treatment), and after finishing abdominal RT (post-treatment).

The glutamine group received $30 \mathrm{~g} / \mathrm{d}$ of oral glutamine (Glutamina NM; Nutrición Médica, Madrid, Spain). This dose of glutamine was selected following previous studies $[12,14]$. The placebo group was given $30 \mathrm{~g} / \mathrm{d}$ of pure whole casein (Proteína NM; Nutrición Médica). Both glutamine and placebo were administered in nonlabeled sachets containing $10 \mathrm{~g}$ of product. 3 sachets/d, were prescribed from 3 days before starting abdominal RT until the end of treatment. Each sachet was dissolved in $200 \mathrm{~mL}$ of water and administered postprandially. Glutamine and placebo were identical in taste, texture and color before and after dissolution. The level of compliance was evaluated by counting the empty sachets retrieved from patients.

The same dose of abdominal RT was given to both groups over 50 days (glutamine $\mathrm{Md}[\mathrm{IQR}]=51.0$ [28.0] Gy; placebo Md [IQR] = 50.4 [29.0] Gy; P = .636), the fractionation dose being $1.8(\mathrm{IQR})=0.1)$ Gy/session for patients receiving glutamine and 1.9 $(\mathrm{IQR})=0.1) \mathrm{Gy} /$ session for those who received placebo $(\mathrm{P}=.745)$.

\section{Ethics statement}

The study followed the Declaration of Helsinki, was evaluated by the Research Ethics Committee of the Complejo Asistencial Universitario of León (CAULE) (March 29, 2011; reference number 1128), and was registered with Clinical Trials (ref. no. NCT00828399). All patients received verbal and written information about the purpose and methodology of the research and were included after signing the informed consent document.

\section{Analytical methods}

Venous blood samples ( $15 \mathrm{~mL})$ were taken using EDTA as an anticoagulant at each visit using Vacutainer (BD, Franklin Lakes, NJ) system from brachiocephalic vein. Total RNA was isolated from whole blood using a RiboPureTM-Blood Kit (Ambion, Paisley, UK) and quantified by spectrophotometry (Nanodrop 1000, Thermo Scientific, Waltham, MA, USA). DNase I (RNase-free) (Ambion, Paisley, UK) was used to remove residual genomic DNA. First-standard complementary DNA (cDNA) was synthesized using High-Capacity cDNA Archive Kit (Applied Biosystems ${ }^{\circledR}$, Paisley, UK) and then, it was 
amplified using TaqMan ${ }^{\circledR}$ Universal PCR Master Mix (Applied Biosystems ${ }^{\circledR}$ ) on a StepOnePlus Real-Time PCR System (Applied Biosystems ${ }^{\circledR}$ ). TaqMan ${ }^{\circledR}$ probes (Table 1) were derived from the commercially available TaqMan ${ }^{\circledR}$ Assays-on-Demand Gene (Applied Biosystems ${ }^{\circledR}$ ). Relative changes in gene expression levels were determined using the 2- $\Delta \Delta C T$ method. The cycle number at which the transcripts were detectable (CT) was normalized to the cycle number of GAPDH detection, referred to as $\triangle \mathrm{CT}$ described previously [15].

\section{Statistical analysis}

Results were expressed as mean values and SEM. Data were compared by analysis of variance and the Newmann Keuls test. Significance was accepted at $\mathrm{p}<0.05$. Values were analyzed using SPSS version 19.0 (IBM Corp, Armonk, NY, USA).

\section{Results}

\section{Patients}

Final analysis was carried out in forty three patients out of the patients included for the pilot trial. Eleven patients dropped out (five glutamine, six placebo). The two groups did not significantly differ in terms of patient characteristics (Table 2). No glutamine related toxicity was reported by any patient included in the study. No difference in body weight change was observed among glutamine and placebo groups.

Table 1. TaqMan probes and GenBank accession numbers of inflammatory and autophagic genes.

\begin{tabular}{lll}
\hline Gene & GenBank accession no. & Probe \\
\hline AP-1 & NM_002228.3 & Hs01103582_s1 \\
Atg5 & NM_004849.2 & Hs00169468_m1 \\
beclin-1 & NM_003766.3 & Hs00186838_m1 \\
CD36 & NM_000072.3 & Hs01567186_m1 \\
COX-2 & NM_000963.2 & Hs00153133_m1 \\
GAPDH & NM_002046.4 & Hs99999905_m1 \\
IL-1 $\beta$ & NM_000576.2 & Hs01555410_m1 \\
LAMP-1 & NM_005561.3 & Hs00174766_m1 \\
LC3-II & NM_003766.3 & Hs00917683_m1 \\
MMP-9 & NM_004994.2 & Hs00234579_m1 \\
p50 & NM_001113511.1 & Hs00388776_m1 \\
P65 & NM_001165412.1 & Hs00765730_m1 \\
p62/SQSTM1 & NM_001142298.1 & Hs01061912_m1 \\
TLR4 & NM_003266.3 & Hs00370853_m1 \\
TNF- $\alpha$ & NM_000594.3 & Hs00174128_m1 \\
UVRAG & NM_003369.3 & Hs00163433_m1
\end{tabular}

Abbreviations: AP-1, activator protein 1; Atg5, autophagy-related protein-5;

COX-2, cyclooxygenase-2; GAPDH, glyceraldehyde-3-phosphate dehydrogenase;

IL-1 $\beta$, interleukin-1 $\beta$. LAMP-1, lysosome-associated membrane protein; LC3-II, protein 1 light chain 3; MMP-9, matrix metalloproteinase-9; p50 and p65 subunits of the nuclear factor kappa B (NF-kB); p62/SQSTM1, sequestosome 1; TLR4, toll-like receptor 4; TNF- $\alpha$, tumor necrosis factor-alpha; UVRAG, UV radiation resistance associated gene.

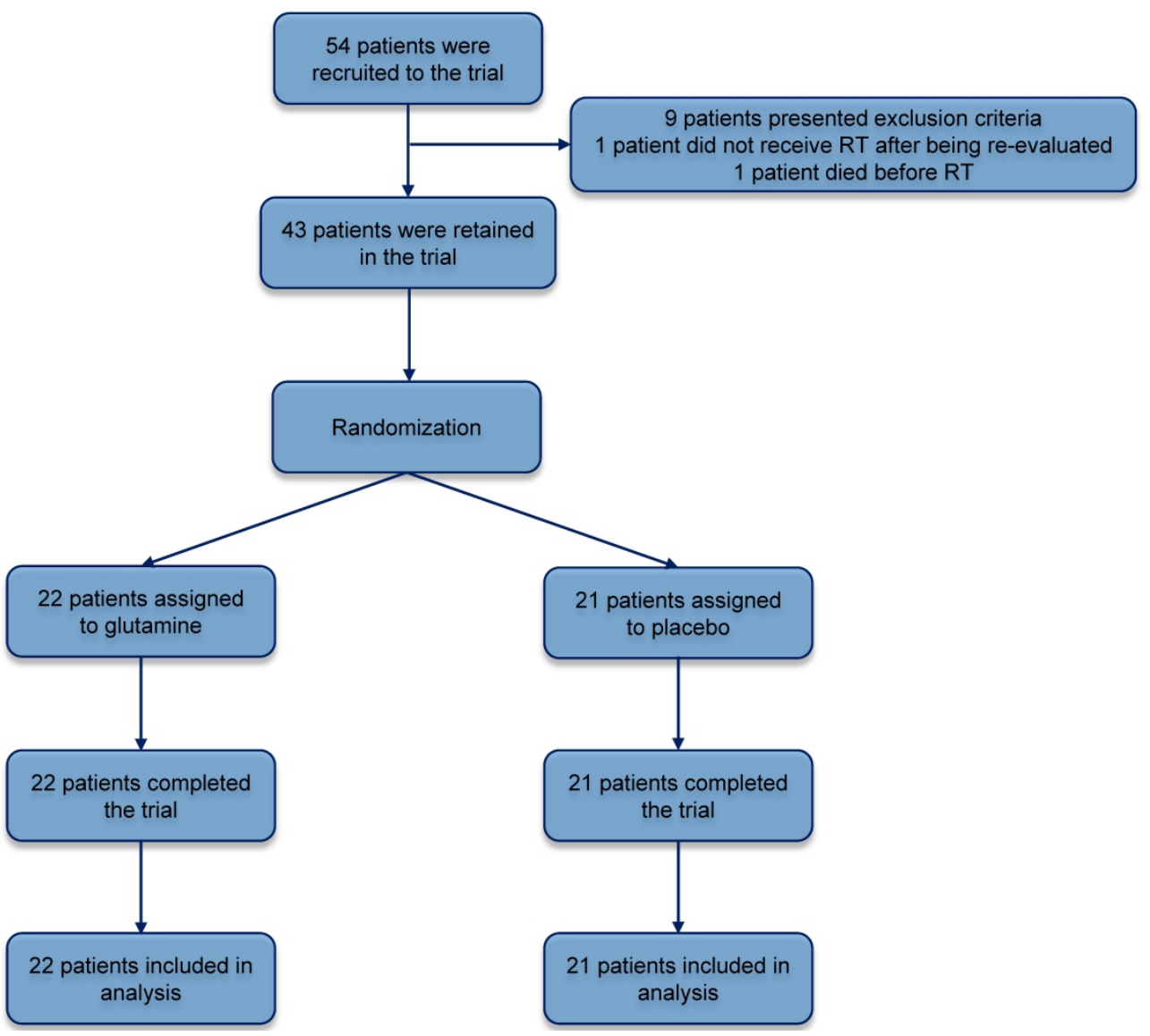

Figure 1. Trial profile; screening, randomization and study completion. RT, radiotherapy. 
Table 2. Patients data

\begin{tabular}{llll}
\hline & $\begin{array}{l}\text { Glutamine } \\
(\mathrm{n}=22)\end{array}$ & $\begin{array}{l}\text { Placebo } \\
(\mathrm{n}=21)\end{array}$ & $p$-value \\
\hline Age, mean (SD) & $66.21(1.71)$ & $68.95(1.75)$ & 0.505 \\
$\begin{array}{l}\text { Male sex, \% } \\
\text { Pathology \% }\end{array}$ & 89.5 & 90.9 & 0.857 \\
Prostate cancer & 68.4 & 59 & 0.112 \\
Rectal cancer & 31.6 & 41 & 0.156 \\
Stage, \% & & & 0.594 \\
I & 0 & 9.1 & \\
II & 68.3 & 54.5 & \\
III & 26.3 & 36.4 & \\
IV & 5.4 & 0 & \\
Chemotherapy, \% & 26.3 & 31.8 & 0.756 \\
Previous surgery, \% & 48.1 & 50.1 & 0.862 \\
\hline
\end{tabular}

Abbreviations: SD, Standard Deviation.

\section{Effects of abdominal RT and glutamine on markers of the inflammatory response}

The effect of abdominal RT and glutamine treatment on mRNA levels of inflammatory markers in whole blood is depicted in Figure 2. TLR4, CD36, IL-1 $\beta$, TNF- $\alpha$, COX-2, and MMP-9 expression increased significantly from the period before treatment to mid-treatment in the placebo group.
Transcripts levels decreased significantly following treatment and those of TLR4, CD36, IL- $1 \beta$, TNF- $\alpha$, and MMP-9 did not differ from values prior to abdominal RT. In the group receiving glutamine, mRNA levels for the different pro-inflammatory genes were significantly lower compared with the placebo group during the mid-treatment period. Consistent with these data, expression of CD36, IL-1 $\beta$, TNF- $\alpha$, and COX-2 did not differ significantly from the period before abdominal RT. During the post-treatment period, mRNA levels of TLR4 were significantly lower in the glutamine group compared with placebo group.

\section{Effects of abdominal RT and glutamine on NF-KB and AP-1 transcription factors}

Results from the patients in the placebo group showed that abdominal RT enhanced the expression of the transcription factors NF- $\mathrm{KB}$ (p50 and p65) and AP1 in the mid-treatment period, with values returning to normal after treatment (Figure 2). Moreover, mRNA levels were significantly lower in the glutamine group compared with placebo during mid-treatment.
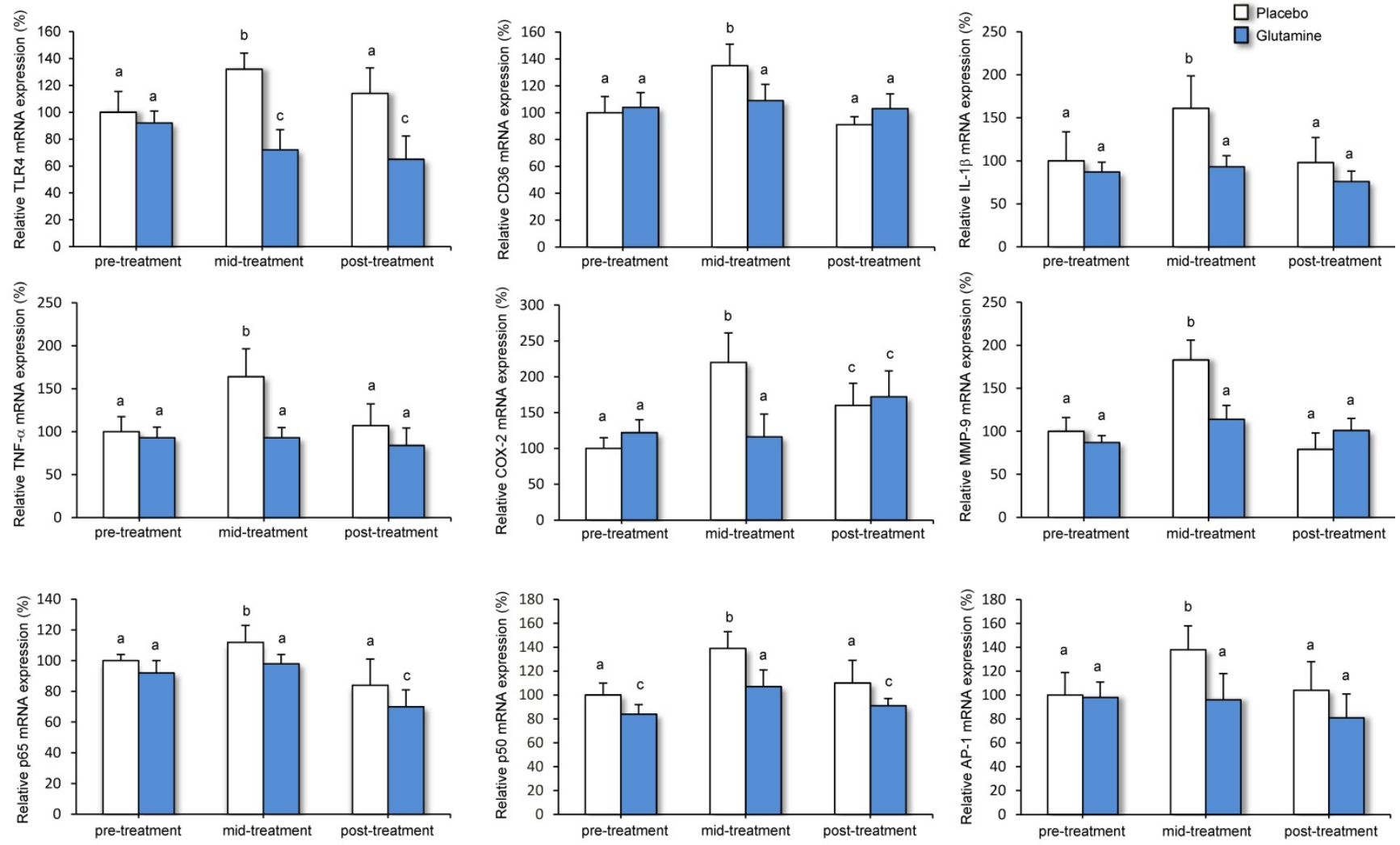

Figure 2. Effect of abdominal RT and treatment with glutamine on mRNA levels of genes related to inflammation in whole blood from cancer patients. Levels of mRNA were analyzed by real-time PCR assays. Data, normalized against GAPDH, are presented as percentage change from pre-treatment values. Means and SEM. a $\mathrm{p}<0.05$, compared with placebo group, same period. $\mathrm{b} p<0.05$, compared with pre-treatment, same group. $c \mathrm{p}<0.05$, compared with placebo and pre-treatment group, same period. 


\section{Effects of abdominal RT and glutamine on markers of autophagic response}

Finally, mRNA expression of different markers of autophagy was measured in whole blood from both placebo and glutamine groups (Figure 3). In the placebo group transcript levels of beclin-1, UVRAG, Atg5, LC3-II, p62 and LAMP-1 were significantly higher in the mid-treatment period compared with the period prior to treatment. Following abdominal RT mRNA levels of beclin-1, UVRAG, Atg5 and LC3-II have returned to pre-treatment levels. When patients were given glutamine, the different autophagy genes showed lower transcript levels compared with the placebo group during the mid-treatment period. P62 and LAMP-1 expression was also significantly lower in glutamine groups compared with placebo group following abdominal RT.

\section{Discussion}

When glutamine falls markedly over time in patients with cancer, glutamine-dependent organs and tissues may be impaired. The extent of radiation damage may be partially prevented in presence of normal glutamine stores. Recent reports indicate that glutamine supplementation minimizes radiation induced dermatitis in breast cancer [16], and decreases the severity of mucositis or esophagitis induced by chemo-radiotherapy in cancer patients with head and neck cancer [8, 17]. Glutamine is essential to maintain a normal gut under radiation. An early inflammatory phase may induce the pathologic progression of radiation toxicity in many tissues. For this reason, a first objective of the present study was to assess the efficacy of oral glutamine in the prevention of radiation-induced inflammation in patients with abdominal cancer.

Subsequent inflammation following microcirculatory breakdown are major features of RT side effects [18]. Early activation of cytokine cascades following radiation triggers different cell responses [19]. Activation of the pro-inflammatory cytokine network by radiation is responsible of multiple symptoms, fatigue, pain, local inflammation, etc, in cancer patients [20]. In our research, expression of the inflammatory markers TLR4, CD36, IL-1 $\beta$, TNF- $\alpha$ and COX-2 increased significantly in the course of abdominal RT, and gene up-regulation was significantly prevented by glutamine administration. These results support previous data indicating that in patients with esophageal cancer immunonutrition with a combination of omega- 3 fatty acids, glutamine, and arginine during concurrent chemoradiotherapy, results in reduced levels of inflammatory cytokines [13].
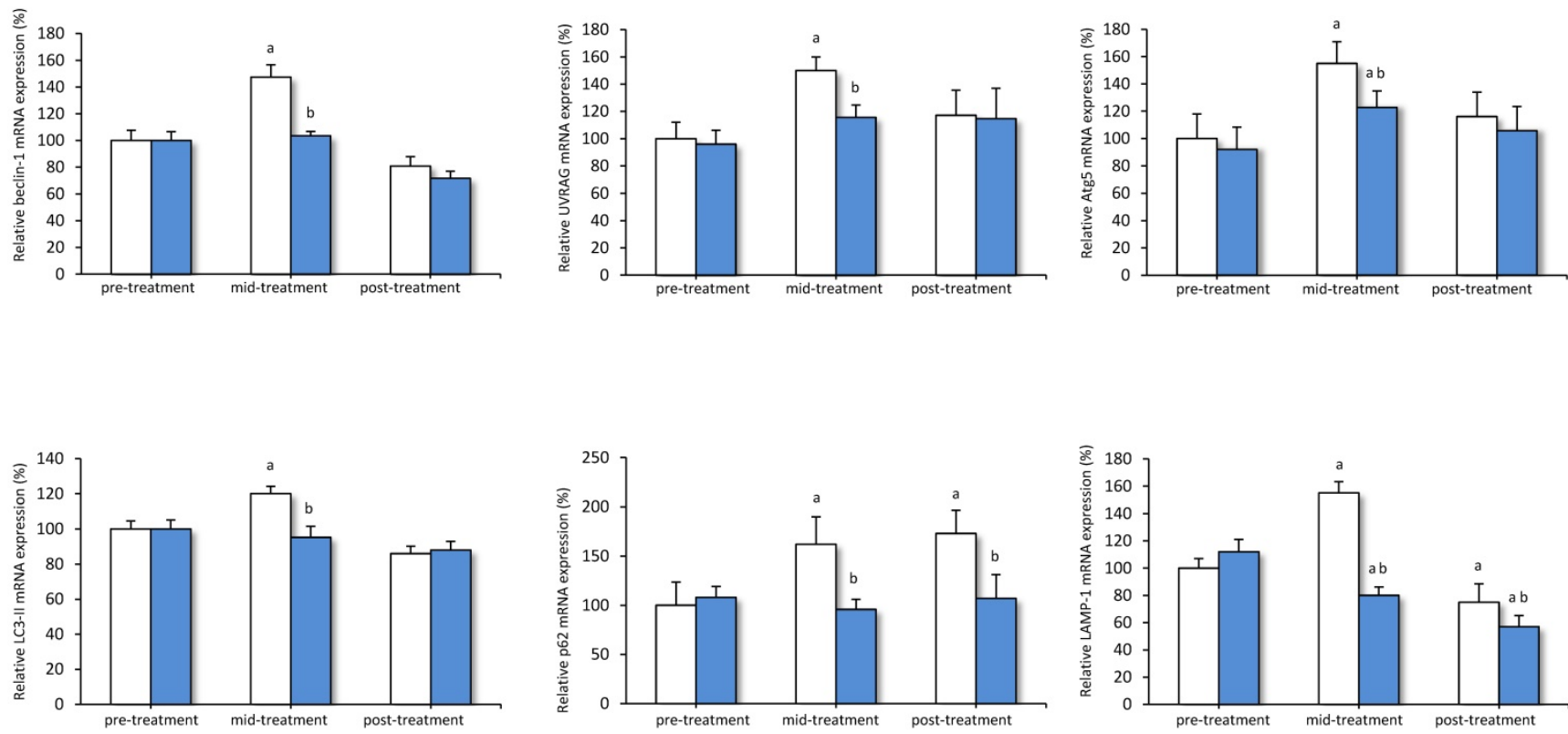

Figure 3. Effect of abdominal RT and treatment with glutamine on mRNA levels of genes related to autophagy in whole blood from cancer patients. Levels of mRNA were analyzed by real-time PCR assays. Data, normalized against GAPDH, are presented as percentage change from pre-treatment values. Means and SEM. a $<<0.05$, compared with placebo group, same period. b $\mathrm{p}<0.05$, compared with pre-treatment, same group. c $\mathrm{p}<0.05$, compared with placebo and pre-treatment group, same period. 
NF- $\mathrm{KB}$ and AP-1 are transcription factors which play critical roles in the generation of cytokines and other molecules involved in the inflammatory response. Activation of NF- $\mathrm{KB}$ pathway by irradiation is known to stimulate inflammatory cytokine expression, whereas activation of AP-1 pathway promotes MMP production [21]. Elevated levels of pro-inflammatory cytokines following RT correlate with histological evidence of intestinal mucositis and peak expression of NF- $\mathrm{KB}$ [22], while antioxidants inhibit irradiation-induced inflammatory cytokine and MMP production by suppressing the NF- $\mathrm{KB}$ and AP-1 signaling [23]. Moreover, it is known that activation of the TLR4/NF- $\mathrm{kB}$ signaling pathway may contribute to radiation resistance, while sensitivity is boosted by the combination of radiotherapy with NF- $\kappa B$ activity-inhibiting drugs [24]. In our study, activation of both AP-1 and NF- $\mathrm{KB}$ resulting from abdominal RT was significantly abolished by glutamine administration, in parallel to TLR4, CD36, MMP-9, and cytokine down-regulation. Taken together data obtained indicate that the application of glutamine could be useful for the weakening of inflammation associated to abdominal RT in cancer patients.

Autophagy is a dynamic pathway responsible for the degradation of organelles and proteins which affects cell metabolism in two opposite ways: either protecting the cell or killing it [4]. Many anticancer agents, tamoxifen, arsenic trioxide, rapamycin, temozolomide, histone deacetylase inhibitors, radiation, vitamin $\mathrm{D}$ analogues, and etoposide have been reported to induce autophagy. However, it is highly controversial whether autophagy has a positive effect in cancer therapy [25]. Autophagy acts in two ways in tumor cells. It may act as a tumor suppressor mechanism, promoting cancer cell proliferation and tumorigenesis, but it may also favor cell survival under oxygen/nutrient deprivation or in response to deleterious effects by chemotherapeutic drugs or ionizing radiation [26].

The role of autophagy in the resistance of cancer cells to RT is still controversial. Nevertheless, it has been shown that pharmacological and/or genetic inhibition of autophagy favors the lethal effects of tumor cells in several therapeutic circumstances. This suggests that intervention in the autophagic pathway may be an alternative in cancer treatment [27]. Inhibition of autophagy has been recently shown to significantly increase both in vitro and in vivo the radiosensitivity of esophageal squamous cell carcinoma [28]. In this context, new autophagy inhibitors that are anticipated to have superior pharmacokinetic properties as well as clinical efficacy are currently under development [29].
Although with the limitation of being a pilot study, this is the first human research to examine the impact of the use of glutamine on autophagy in cancer patients treated with RT. The autophagosome is a double-membrane-bound vacuole which engulfs fractions of the cytoplasm through different autophagy adaptors, such as p62/SQSTM1. This mechanism results in the autophagic degradation of ubiquitinated protein aggregates in lysosomes. The first step in the autophagic process, the nucleation of the autophagosomal membrane, is under the control of a molecular complex containing beclin-1. Several interacting proteins participating in this complex include positive factors such as UVRAG. The elongation step requires two distinct ubiquitin-like conjugation systems. The first system involves conjugation of Atg12 to Atg5. In the second conjugation system LC3-I conjugates with phosphatidylethanolamine to LC3-II, which localizes to the autophagosomal membrane [4]. Data from the present study indicate that RT coursed with an increase in beclin-1 mRNA levels in parallel to changes in both ubiquitine systems (Atg and LC3-II). This effect was associated with an increased expression of UVRAG, which interacts with beclin-1 in the early steps. Autophagosome maturation would subsequently activate autophagy.

These results should be analyzed with caution. LC3 protein may accumulate as a result of either higher autophagic flux by increased formation and processing of autophagosomes or blocking of autophagy caused by an absence of autophagolysosomal processing [30]. In the present research the lysosomal marker LAMP-1 was also increased by abdominal RT, which suggests reduced lysosomal consumption. In addition, the higher expression of p62/SQSTM1 could be associated to deceleration of recycling of the major carrier of material to autophagosomes and a lower autophagic degradation of ubiquitinated protein aggregates in lysosomes [31]. In any case, all the described effects of abdominal RT on the different autophagy markers were significantly abrogated by glutamine. These open the possibility to further studies aimed to investigate whether glutamine actions on autophagy induced by radiation can be of therapeutic benefit.

\section{Conclusions}

In summary, the present results shed new light into the role of glutamine in human, indicating that it decreases the expression of inflammatory mediators during abdominal RT-induced healing response and abolishes autophagic changes associated with RT. Identification of glutamine actions may contribute to a better understanding of its use in cancer patients. 


\section{Abbreviations}

AP-1: Activator protein 1; Atg5: Autophagyrelated protein-5; COX-2: Cyclooxygenase-2; IL-1 $\beta$ : Interleukin 1 $1 \beta$ LAMP-1: Lysosome-associated membrane protein; LC3: Protein 1 light chain 3; MMP-9: Matrix metalloproteinase-9; NF-кB: Nuclear factor kappa B; p62/SQSTM1: Sequestosome 1; RT: Radiotherapy; TLR4: Toll-like receptor 4; TNF- $\alpha$ : Tumor necrosis factor-alpha; UVRAG: UV radiation resistance associated gene.

\section{Acknowledgements}

This work has been partially supported by the Gerencia Regional de Salud de Castilla y León (grant GRS 638-A-11), Spain. CIBEREHD is funded by Instituto de Salud Carlos III. D.I. Sánchez is granted by AECC.

\section{Competing Interests}

The authors have declared that no competing interest exists.

\section{References}

1. Linard C, Ropenga A, Vozenin-Brotons MC, Chapel A, Mathe D. Abdominal irradiation increases inflammatory cytokine expression and activates NF-kappaB in rat ileal muscularis layer. Am J Physiol Gastrointest Liver Physiol. 2003;285:G556-65.

2. Ong ZY, Gibson RJ, Bowen JM, Stringer AM, Darby JM, Logan RM, et al. Pro-inflammatory cytokines play a key role in the development of radiotherapy-induced gastrointestinal mucositis. Radiat Oncol. 2010;5:22.

3. O'Neil BH, Funkhouser WK, Calvo BF, Meyers MO, Kim HJ, Goldberg RM, et al. Nuclear factor $\kappa$-light chain-enhancer of activated B cells is activated by radiotherapy and is prognostic for overall survival in patients with rectal cancer treated with preoperative fluorouracil-based chemoradiotheraphy. Int J Radiat Oncol Biol Phys. 2011;80:705-11.

4. Vallejo D, Crespo I, San-Miguel B, Álvarez M, Prieto J, Tuñón MJ, et al. Autophagic response in the rabbit hemorrhagic disease, an animal model of virally-induced fulminant hepatic failure. Vet Res. 2014;4:15.

5. Zois CE, Koukourakis MI. Radiation-induced autophagy in normal and cancer cells: towards novel cytoprotection and radio-sensitization policies? Autophagy. 2009;5:442-50.

6. Amaravadi RK, Yu D, Lum JJ, Bui T, Christophorou MA, Evan GI, et al Autophagy inhibition enhances therapy-induced apoptosis in a Myc-induced model of lymphoma. J Clin Invest. 2007;117:326-36.

7. Ko A, Kanehisa A, Martins I, Senovilla L, Chargari C, Dugue D, et al. Autophagy inhibition radiosensitizes in vitro, yet reduces radioresponses in vivo due to deficient immunogenic signalling. Cell Death Differ. 2014;21:92-9. doi: $10.1038 /$ cdd.2013.124.

8. Gul K, Muge A, Taner A, Sehri E. Oral glutamine supplementation reduces radiotherapy induced esophagitis in lung cancer patients. Asian Pac J Cancer Prev. 2015;16:53-8.

9. Huang EY, Leung SW, Wang CJ, Chen HC, Sun LM, Fang FM, et al. Oral GLN to alleviate radiation-induced oral mucositis: A pilot randomized trial. Int J Radiat. Oncol. Biol Phys. 2000;46:535-9.

10. Vidal-Casariego A, Calleja-Fernández A, de Urbina-González JJ, Cano-Rodríguez I, Cordido F, Ballesteros-Pomar MD. Efficacy of glutamine in the prevention of acute radiation enteritis: a randomized controlled trial. JPEN J Parenter Enteral Nutr. 2014;38:205-13.

11. Ramirez Vargas R. Efficacy of glutamine prophylactic treatment on enteritis in patients due to abdominal and pelvic radiation therapy. Radiother Oncol. 2009;90:S27.

12. Vidal-Casariego A, Calleja-Fernández A, Cano-Rodríguez I, Cordido F, Ballesteros-Pomar MD. Effects of oral glutamine during abdominal radiotherapy on chronic radiation enteritis: a randomized controlled trial. Nutrition. 2015;31:200-4.

13. Sunpaweravong $\mathrm{S}$, Puttawibul $\mathrm{P}$, Ruangsin $\mathrm{S}$, Laohawiriyakamol $\mathrm{S}$, Sunpaweravong P, Sangthawan D, et al. Randomized study of antiinflammatory and immune-modulatory effects of enteral immunonutrition during concurrent chemoradiotherapy for esophageal cancer. Nutr Cancer. 2014;66:1-5.
14. Sayles C, Hickerson SC, Bhat RR, Hall J, Garey KW, Trivedi MV. Oral glutamine in preventing treatment-related mucositis in adult patients with cancer: a systematic review. Nutr Clin Pract. 2016;31: 171-9.

15. Crespo I, García-Mediavilla MV, Almar M, González P, Tuñón MJ, Sánchez-Campos S, et al. Differential effects of dietary flavonoids on reactive oxygen and nitrogen species generation and antioxidant defenses in Chang Liver cells. Food Chem Toxicol. 2008;46: 1555-69.

16. Eda K, Uzer K, Murat T, Cenk U. The effects of enteral glutamine on radiotherapy induced dermatitis in breast cancer. Clin Nutr. 2016;35:436-9.

17. Leung HW, Chan AL. Glutamine in alleviation of radiation-induced severe oral mucositis: a meta-analysis. Nutr Cancer. 2016;4:1-9.

18. Goertz O, Poettgen C, Akbari A, Kolbenschlag J, Langer S, Lehnhardt M, et al. New model for long-term investigations of cutaneous microcirculatory and inflammatory changes following irradiation. J Radiat Res. 2015;56:456-61.

19. Bentzen SM. Preventing or reducing late side effects of radiation therapy: radiobiology meets molecular pathology. Nat Rev Cancer. 2006, 6:702-13. doi:10.1038/nrc1950.

20. Deorukhkar A, Krishnan S. Targeting inflammatory pathways for tumor radiosensitization. Biochem Pharmacol. 2010;80:1904-14.

21. Vicentini FT, He T, Shao Y, Fonseca MJ, Verri WA Jr, Fisher GJ, et al. Quercetin inhibits UV irradiation-induced inflammatory cytokine production in primary human keratinocytes by suppressing NF- $\mathrm{KB}$ pathway. J Dermatol Sci. 2011;61:162-8.

22. Yeoh AS, Gibson RJ, Yeoh EE, Bowen JM, Stringer AM, Giam KA, et al. A novel animal model to investigate fractionated radiotherapy-induced alimentary mucositis: the role of apoptosis, p53, nuclear factor-kappaB, COX-1, and COX-2. Mol Cancer Ther. 2007:6:2319-27.

23. Kim SR, Jung YR, An HJ, Kim DH, Jang EJ, Choi YJ, et al. Anti-wrinkle and anti-inflammatory effects of active garlic components and the inhibition of MMPs via NF- $\kappa B$ signaling. PLoS One. 2013;8:e73877.

24. Tang H, Wang F, Zhou XF, Zhou J, Chen L, Luo JD, et al. Relationship between TLR4 and NF-KB p65 protein expressions and clinical radiosensitivity of patients with esophageal squamous cell carcinoma. Pak J Med Sci. 2014;30:982-5.

25. Sharma K, Le N, Alotaibi M, Gewirtz DA. Cytotoxic autophagy in cancer therapy. Int J Mol Sci. 2014;15:10034-51.

26. Rabinowitz JD, White E. Autophagy and metabolism. Science. 2010;330:1344-8.

27. Choi KS. Autophagy and cancer. Exp Mol Med. 2012;44:109-20.

28. Chen Y, Li X, Guo L, Wu X, He C, Zhang S, et al. Combining radiation with autophagy inhibition enhances suppression of tumor growth and angiogenesis in esophageal cancer. Mol Med Rep. 2015;12:1645-52.

29. Sehgal AR, Konig H, Johnson DE, Tang D, Amaravadi RK, Boyiadzis M, et al. You eat what you are: autophagy inhibition as a therapeutic strategy in leukemia. Leukemia. 2015;29:517-25.

30. Klionsky DJ, Abdalla FC, Abeliovich H, Abraham RT, Acevedo-Arozena A, Adeli $\mathrm{K}$, et al. Guidelines for the use and interpretation of assays for monitoring autophagy. Autophagy. 2012;8:445-544.

31. Kalamida D, Karagounis IV, Giatromanolaki A, Koukourakis MI. Important role of autophagy in endothelial cell response to ionizing radiation. PLoS One. 2014;9: e102408 